\title{
ON THE GENERALIZED WAZEWSKI INEQUALITY FOR RETARDED SYSTEMS
}

\section{I. Gil'}

\section{Introduction and statement of the main result}

Let us consider in a Euclidean space $\mathbf{C}^{n}$ with the Euclidean norm $\|\cdot\|_{C^{n}}$ the equation

$$
\dot{x}=A(t) x, \quad \dot{u}=\frac{d u}{d t}, \quad t \geq 0,
$$

where $A(t)$ is a variable matrix. Denote by $(\cdot, \cdot)$ the scalar product in $\mathbf{C}^{n}$. As is well known, Wazewski [6] established the very useful inequality

$$
\left\|U_{A}(t, s)\right\|_{C^{n}} \leq e^{\int_{s}^{t} \alpha(A(\tau)) d \tau}, \quad t, s \geq 0
$$

where $U_{A}(t, s)$ is the evolution operator of the equation (1.1) and

$$
\alpha(A(t))=\sup _{x \in \mathbf{C}^{n}} \operatorname{Re}(A(t) x, x)(x, x)^{-1}
$$

(see also Winter [7], Daleckii and Krein [1, Section 3.4.6]).

The present paper is devoted to a generalization of inequality (1.2) to the equation

$$
\dot{x}(t)=A(t) x(t)+B(t) x(t-h), \quad t \geq 0, h=\text { const. }>0
$$

where $B(t)$ is a variable matrix. Note that an estimate for the Lyapunov exponents of equation (1.3) is presented in Kolmanovskii [3], among other interesting results. Under some restrictions below, we make the Kolmanovskii result more precise. In the scalar case, our results supplement the well-known stability conditions from Yoneyama [8].

Take the initial condition

$$
x(t)=\Phi(t) \quad \text { for }-h \leq t \leq 0
$$

where $\Phi(t)$ is a given continuous vector-valued function. A solution of (1.3) is an absolutely continuous function $x(t):[-h, \infty) \rightarrow \mathbf{C}^{n}$ which satisfies that equation on $[0, \infty)$ almost everywhere with a given initial condition (cf. Hale and Verduyn Lunel [2], Kolmanovskii and Myshkis [4]). The existence of solutions is assumed.

Let us suppose that the functions $\alpha(A(t))$ and

$$
\alpha(B(t)) \equiv \sup _{x \in \mathbf{C}^{n}} \operatorname{Re}(B(t) x, x)(x, x)^{-1}
$$

are Riemann integrable. Put

$$
\alpha_{W}(t)=\alpha(A(t))+\alpha(B(t+h)) .
$$

The aim of the present paper is to prove the following:

Received August 6, 1997, revised April 30, 1998.

1991 Mathematics Subject Classification: 34K20.

Key words and phrases: linear retarded systems, stability. 
Theorem 1.1. Let the inequality

$$
J_{B} \equiv \sup _{t \geq 0} \int_{t}^{t+h}\|B(s)\|_{C^{n}} d s<1
$$

be fulfilled. Then for any solution $x(t)$ of (1.3) with a continuous initial function $\Phi(t)$, the estimate

$$
\|x(t)\|_{C^{n}} \leq\left(1-J_{B}\right)^{-1} c_{W}(\Phi) \exp \left[\int_{0}^{t}\left(\alpha_{W}(s)+\eta_{W}(s)\right) d s\right], \quad t \geq h,
$$

is valid with the notation

$$
\eta_{W}(s)=\left(1-J_{B}\right)^{-1}\|B(s+h)\|_{C^{n}} \int_{s}^{s+h}\|A(\tau)+B(\tau+h)\|_{C^{n}} e^{\int_{\tau}^{s} \alpha_{W}\left(\tau_{1}\right) d \tau_{1}} d \tau
$$

and

$$
c_{W}(\Phi)=\|\Phi(0)\|_{C^{n}}+\int_{0}^{h} e^{-\int_{0}^{s} \alpha_{W}(\tau) d \tau}\|B(s) \Phi(s-h)\|_{C^{n}} d s .
$$

If $B(t) \equiv 0$, then (1.6) coincides with the Wazewski inequality (1.2).

\section{Proof of Theorem 1.1}

Put $\tilde{A}(t)=A(t)+B(t+h)$ and denote by $U(t, s)$ the evolution operator of the equation

$$
\dot{x}(t)=\tilde{A}(t) x(t) .
$$

Let us suppose that there is a Riemann integrable function $\alpha(t)$ and a positive constant $K$ such that

$$
\|U(t, s)\|_{C^{n}} \leq K e^{\int_{s}^{t} \alpha(\tau) d \tau}, \quad t, s \geq 0 .
$$

Lemma 2.1. Let the inequalities (2.1) and

$$
\theta \equiv K \sup _{t \geq 0} \int_{t}^{t+h}\|B(s)\|_{C^{n}} d s<1
$$

be fulfilled. Then for any solution $x(t)$ of (1.3) with a continuous initial function $\Phi(t)$, the estimate

$$
\|x(t)\|_{C^{n}} \leq(1-\theta)^{-1} c(\Phi) \exp \left[\int_{0}^{t}(\alpha(s)+\eta(s)) d s\right], \quad t \geq h,
$$

is valid with the notation

$$
\eta(s)=K(1-\theta)^{-1}\|B(s+h)\|_{C^{n}} \int_{s}^{s+h}\|A(\tau)+B(\tau+h)\|_{C^{n}} e^{\int_{\tau}^{s} \alpha\left(\tau_{1}\right) d \tau_{1}} d \tau
$$

and

$$
c(\Phi)=K\left[\|\Phi(0)\|_{C^{n}}+\int_{0}^{h} e^{-\int_{0}^{s} \alpha(\tau) d \tau}\|B(s) \Phi(s-h)\|_{C^{n}} d s\right] .
$$

Proof. For the sake of simplicity, throughout this proof, we set $\|\cdot\|_{C^{n}}=|\cdot|$. Rewrite (1.3) in the form

$$
\dot{x}(t)=\tilde{A}(t) x(t)+B(t) x(t-h)-B(t+h) x(t), \quad t \geq 0 .
$$


This equation is equivalent to the following:

$$
x(t)=U(t, 0) x(0)+\int_{0}^{t} U(t, s)[B(s) x(s-h)-B(s+h) x(s)] d s, \quad t \geq 0 .
$$

Hence,

$$
\begin{gathered}
x(t)=U(t, 0) x(0)+\int_{0}^{t-h}[U(t, s+h)-U(t, s)] B(s+h) x(s) d s \\
-\int_{t-h}^{t} U(t, s) B(s+h) x(s) d s+f_{1}(t), \quad t \geq h,
\end{gathered}
$$

where

$$
f_{1}(t)=\int_{0}^{h} U(t, s) B(s) \Phi(s-h) d s
$$

By (2.1), we get

$$
\left|\int_{t-h}^{t} U(t, s) B(s+h) x(s) d s\right| \leq K \int_{t-h}^{t} e^{\int_{s}^{t} \alpha(\tau) d \tau}|B(s+h)||x(s)| d s .
$$

Further, it can be written,

$$
\begin{aligned}
\tilde{J}(t) & \equiv\left|\int_{0}^{t-h}[U(t, s+h)-U(t, s)] B(s+h) x(s) d s\right| \\
& \leq \int_{0}^{t-h}|U(t, s+h)-U(t, s)||B(s+h)||x(s)| d s .
\end{aligned}
$$

Take into account that

$$
\frac{\partial}{\partial s} U(t, s)=-U(t, s) \tilde{A}(s)
$$

(cf. Tanabe [5, p. 89]). Then, by (2.1),

$$
\begin{aligned}
|U(t, s+h)-U(t, s)| & \leq \int_{s}^{s+h}|U(t, \tau) \tilde{A}(\tau)| d \tau \\
& \leq e^{\int_{s}^{t} \alpha\left(\tau_{1}\right) d \tau_{1}} K \int_{s}^{s+h} e^{\int_{\tau}^{s} \alpha\left(\tau_{1}\right) d \tau_{1}}|\tilde{A}(\tau)| d \tau
\end{aligned}
$$

Therefore,

$$
\tilde{J}(t) \leq \int_{0}^{t-h} e^{\int_{s}^{t} \alpha(\tau) d \tau} w(s)|x(s)| d s
$$

where

$$
w(s)=K|B(s+h)| \int_{s}^{s+h} e^{\int_{\tau}^{s} \alpha\left(\tau_{1}\right) d \tau_{1}}|\tilde{A}(\tau)| d \tau .
$$

In addition, thanks to (2.1),

$$
\begin{aligned}
\left|U(t, 0) x(0)+f_{1}(t)\right| & \leq K\left[e^{\int_{0}^{t} \alpha(\tau) d \tau}|x(0)|+\int_{0}^{h} e^{\int_{s}^{t} \alpha(\tau) d \tau}|B(s) \Phi(s-h)| d s\right] \\
& =e^{\int_{0}^{t} \alpha(\tau) d \tau} c(\Phi) .
\end{aligned}
$$


Now (2.2)-(2.4) imply

$$
\begin{gathered}
|x(t)| \leq e^{\int_{0}^{t} \alpha(\tau) d \tau} c(\Phi)+\int_{0}^{t-h} e^{\int_{s}^{t} \alpha(\tau) d \tau} w(s)|x(s)| d s \\
+K \int_{t-h}^{t} e^{\int_{s}^{t} \alpha(\tau) d \tau}|B(s+h)||x(s)| d s
\end{gathered}
$$

Put

$$
y(t)=|x(t)| e^{-\int_{0}^{t} \alpha(\tau) d \tau} .
$$

Clearly,

$$
y(t) \leq c(\Phi)+K \int_{t-h}^{t}|B(s+h)| y(s) d s+\int_{0}^{t-h} w(s) y(s) d s
$$

Denote

$$
\zeta(t)=\max _{0 \leq s \leq t} y(s) .
$$

Then, for a fixed positive $T<\infty$,

$$
\zeta(T) \leq c(\Phi)+\theta \zeta(T)+\int_{0}^{T-h} w(s) \zeta(s) d s
$$

Under the condition $\theta<1$, this gives

$$
\zeta(T) \leq(1-\theta)^{-1}\left[c(\Phi)+\int_{0}^{T} w(s) \zeta(s) d s\right] .
$$

Replacing $T$ by $t$ and applying the Gronwall inequality, we get

$$
\zeta(t) \leq(1-\theta)^{-1} c(\Phi) \exp \left[(1-\theta)^{-1} \int_{0}^{t} w(s) d s\right] .
$$

Since $y(t) \leq \zeta(t)$, due to (2.5), we arrive at the required estimate.

Proof of Theorem 1.1. The result is due to (1.2) and Lemma 2.1.

\section{Stability conditions}

Theorem 1.1 yields

Corollary 3.1. Let the inequalities (1.5) and

$$
\varlimsup_{t \rightarrow \infty} \int_{0}^{t}\left[\alpha_{W}(s)+\eta_{W}(s)\right] d s<\infty
$$

be fulfilled. Then equation (1.3) is stable.

Let us assume that under the condition $\alpha_{W}(t) \leq 0, t \geq 0$, the inequalities

$$
\begin{aligned}
J_{A+B} & \equiv \sup _{t \geq 0} \int_{t}^{t+h}\|A(s)+B(s+h)\|_{C^{n}} d s<\infty, \\
\Lambda_{W} & \equiv \sup _{t \geq 0} \int_{t}^{t+h}\left|\alpha_{W}(s)\right| d s<\infty
\end{aligned}
$$

are fulfilled. Then clearly,

$$
\int_{0}^{t} \eta_{W}(s) d s \leq J_{A+B}\left(1-J_{B}\right)^{-1} e^{\Lambda_{W}} \int_{0}^{t}\|B(s+h)\|_{C^{n}} d s .
$$


Further, take into account that

$$
\begin{aligned}
\varlimsup_{t \rightarrow \infty} t^{-1} \int_{0}^{t}\|B(s+h)\|_{C^{n}} d s & \leq \varlimsup_{j \rightarrow \infty}(h j)^{-1} \int_{0}^{(j+1) h}\|B(s+h)\|_{C^{n}} d s \\
& \leq \varlimsup_{j \rightarrow \infty}(h j)^{-1} \sum_{k=0}^{j} \int_{k h}^{(k+1) h}\|B(s+h)\|_{C^{n}} d s \leq h^{-1} J_{B} .
\end{aligned}
$$

Now Corollary 3.1 implies

Corollary 3.2. Under conditions (1.5) and (3.2), let the inequality

$$
\varlimsup_{t \rightarrow \infty} t^{-1} \int_{0}^{t} \alpha_{W}(s) d s+\frac{e^{\Lambda_{W}} J_{B} J_{A+B}}{h\left(1-J_{B}\right)} \leq 0
$$

be fulfilled. Then equation (1.3) is stable.

If the inequality

$$
\int_{t_{0}}^{t}\left[\alpha(B(s+h))+\eta_{W}(s)\right] d s \leq \int_{t_{0}}^{t}\|B(s)\|_{C^{n}} d s \quad t \geq t_{0}
$$

holds, then Corollary 3.1 under (1.5) makes the following stability conditions more precise:

$$
\int_{t_{0}}^{t}\left[\alpha(A(s))+\|B(s)\|_{C^{n}}\right] d s \leq 0, \quad t \geq t_{0},
$$

from Kolmanovski [3]. Consider now the scalar equation

$$
\dot{x}(t)=-b(t) x(t-h)
$$

with a continuous positive function $b(t)$. Denote by $z_{0}$ the unique real root of the equation $z\left(1+e^{z}\right)=1$. Clearly, $0<z_{0}<1$. Assume that

$$
J_{B}=\sup _{t \geq 0} \int_{t}^{t+h} b(s) d s<z_{0} .
$$

Then $J_{B}<1$, and $J_{B}\left(1+e^{J_{B}}\right)<1$, since $z\left(1+e^{z}\right)$ increases as $z>0$ increases. Therefore,

$$
\left(1-J_{B}\right)^{-1} J_{B} e^{J_{B}}<1
$$

On the other hand,

$$
\begin{aligned}
\eta_{W}(s) & =\left(1-J_{B}\right)^{-1} b(s+h) \int_{s}^{s+h} b(\tau+h) e^{-\int_{\tau}^{s} b\left(\tau_{1}+h\right) d \tau_{1}} d \tau \\
& \leq\left(1-J_{B}\right)^{-1} b(s+h) J_{B} e^{J_{B}}
\end{aligned}
$$

Relation (3.5) implies

$$
\begin{aligned}
\int_{0}^{t}\left[\eta_{W}(s)+\alpha_{W}(s)\right] d s & \leq \int_{0}^{t}\left[-b(s+h)+J_{B} e^{J_{B}}\left(1-J_{B}\right)^{-1} b(s+h)\right] d s \\
& \leq\left(-1+J_{B} e^{J_{B}}\left(1-J_{B}\right)^{-1}\right) \int_{0}^{t} b(s+h) d s<0, \quad t \geq 0
\end{aligned}
$$

So condition (3.1) holds, and the following result is true:

Corollary 3.3. Let inequality (3.4) be fulfilled. Then equation (3.3) is stable. 
This result supplements the " $3 / 2$-stability theorem" (Yoneyama [8]), which gives for equation (3.3) the following stability condition:

$$
h \sup _{t} b(t) \leq \frac{3}{2}
$$

Finally, note that Theorem 1.1 can be easily extended to the equation

$$
\dot{x}(t)=\sum_{k=0}^{m} A_{k}(t) x\left(t-h_{k}\right) \quad t \geq 0
$$

where $A_{k}(t), k=0, \ldots, m$, are variable matrices, and $h_{0}, \ldots, h_{m}$ are positive numbers.

\section{References}

1. Yu L. Daleckii and M. G. Krein, Stability of Solutions of Differential Equations in Banach Space, Amer. Math. Soc., Providence, R. I., 1974.

2. J. K. Hale and S. M. Verduyn Lunel, Introduction to Functional Differential Equations, SpringerVerlag, 1993.

3. V. Kolmanovskii, (1996). Applications of differential inequalities for stability of some functional differential equations, Nonlinear Analysis, TMA (1996), 1017-1028.

4. V. Kolmanovskii and A. Myshkis, Applied Theory of Functional Differential Equations, Kluwer Academic Publishers, London, 1992.

5. H. Tanabe, Equations of Evolution, Pitman, London, 1979.

6. T. Wazewski, Sur la limitationdes integrales d'equations differentialies linearies ordinaires, Studia. Matem. 59 (1948), 48-59.

7. A. Winter, Asymptotic integration constants, Amer. J. Math. 68 (1946), 125-132

8. T. Yoneyama, The 3/2-stability theorem for one dimensional delay-differential equations, J. of Math. Anal. Appl. 125 (1987), 161-173.

Department of Mathematics, Ben Gurion University of the Negev, P.0. Box 653, BeerSHEVA 84105, ISRAEL

E-mail: gilmi@cs.bgu.ac.il 